\title{
Predictive role of altered leptin, adiponectin and 3-carboxy-4-methyl-5-propyl-2-furanpropanoic acid secretion in gestational diabetes mellitus
}

\author{
ANDREEA ROXANA FLORIAN ${ }^{1}$, GHEORGHE CRUCIAT ${ }^{1}$, RALUCA MARIA POP ${ }^{2}$, \\ ADELINA STAICU $^{1}$, MURESAN DANIEL $^{1}$ and STAMATIAN FLORIN ${ }^{1}$

\begin{abstract}
${ }^{1}$ Obstetrics and Gynecology I, Mother and Child Department, 'Iuliu Hațieganu' University of Medicine and Pharmacy, 400006 Cluj-Napoca; ${ }^{2}$ Department of Pharmacology, Toxicology and Clinical Pharmacology,

'Iuliu Hațieganu' University of Medicine and Pharmacy, 400337 Cluj-Napoca, Romania
\end{abstract}

Received December 24, 2020; Accepted January 26, 2021

DOI: $10.3892 /$ etm.2021.9951

\begin{abstract}
Gestational diabetes mellitus (GDM) is one of the most common complications of pregnancy, leading to considerable maternal and fetal risks. The main aim of this study was to determine the predictive value of the levels of adiponectin (AN), leptin (L) and CMPF (3-carboxy-4-methyl-5-propyl-2-furanpropanoic acid) in the development of GDM. We conducted a prospective longitudinal study on 68 pregnant women that were not at risk of developing GDM, in whom we determined AN, L, CMPF levels at 11-13 weeks +6 days of pregnancy during the first trimester screening. Twenty-one of all the patients included in the study developed GDM during pregnancy. Oral glucose tolerance test (OGTT)/75 g was performed at 24-28 weeks of gestation. L levels were significantly higher in patients who developed GDM than in those who did not develop diabetes $(\mathrm{P}<0.001)$. The $\mathrm{AN} / \mathrm{L}$ ratio was significantly lower in patients with GDM $(\mathrm{P}=0.03)$. AN and $\mathrm{CMPF}$ levels were not associated with GDM. The probability of developing gestational diabetes was higher in patients with L levels above the $\mathrm{L}$ cut-off value of $16 \mathrm{ng} / \mathrm{ml}$ [area under the curve (AUC), 0.775; 95\% confidence interval (CI) $0.658-0.867$ ], sensitivity $100 \%$ (95\% CI 83.9-100), specificity $48.9 \%$ (95\% CI 34.1-63.9) $(\mathrm{P}<0.001)$. Advanced maternal age and higher L levels were
\end{abstract}

Correspondence to: Dr Gheorghe Cruciat, Obstetrics and Gynecology I, Mother and Child Department, 'Iuliu Hațieganu' University of Medicine and Pharmacy, 3-5 Clinicilor Street, 400006 Cluj-Napoca, Romania

E-mail: cruciat@yahoo.com

Abbreviations:CMPF,3-carboxy-4-methyl-5-propyl-2-furanpropanoic acid; GDM, gestational diabetes mellitus; AKs, adipokines; AN/L, adiponectin/leptin ratio; OGTT, oral glucose tolerance test; BMI, body mass index; WG, weeks of gestation; PROM, premature rupture of membranes

Key words: leptin, adiponectin, CMPF, adiponectin/leptin ratio, gestational diabetes mellitus, adipokines found to be predictive factors [odds ratio $(\mathrm{OR})=1.16$ and $\mathrm{OR}=1.06$, respectively] independently associated with gestational diabetes. In as far as general factors are concerned, the patient BMI (body mass index) at the beginning of the pregnancy and smoking were found to be the main risk factors for the onset of GDM. This study showed that elevated L levels are a strong predictor of GDM, while AN and CMPF levels are not, as they failed to show a significant association.

\section{Introduction}

Gestational diabetes mellitus (GDM), defined as glucose intolerance that develops or is diagnosed during pregnancy, is one of the most common complications of pregnancy, leading to considerable maternal-fetal risks (1). There has been an increase in the prevalence of GDM worldwide, as a consequence of the increase in the frequency of type 2 diabetes mellitus (T2DM) and maternal obesity. Patients with GDM are 2-3 times more likely to develop T2DM throughout their lifetime, a pathology that is the seventh leading cause of death worldwide, and whose treatment requires numerous resources (2).

The International Association of Diabetes and Pregnancy Study Groups (IADPSG) report an average worldwide incidence rate of $17.8 \%$ for GDM (between 9.3-25.5\%) (3).

GDM is known to negatively influence pregnancy as early as the first trimester; the frequency of malformations being higher in patients with GDM than in the general population. The most frequent anomalies are cardiovascular and neural tube defects (4). Therefore, an early diagnosis of GDM would help improve maternal-fetal prognosis (5).

Current trends in therapeutic management aim at the discovery of biomarkers for the early detection of GDM or a better selection of the patients at risk. Adipokines (AKs) have also been studied in this regard, and adiponectin (AN) and leptin (L) have been reported to play an important part in the early detection of GDM. AKs are proteins secreted by adipocytes that interfere with the glycoregulatory processes and are involved in numerous other endocrine and metabolic processes such as insulin secretion, insulin resistance 
regulation, as well as a number of inflammatory processes, and body weight regulation (6). Studies have shown abnormal regulation of AKs at the placental level in patients with GDM, due to a low expression of AN (7).

Metabolomics, that can identify metabolites resulting from biochemical reactions, is yet another promising direction of research attempting to explain the pathophysiology of GDM. These metabolites cause subtle metabolic changes in human fluids and tissues and may be involved in the development of GDM. 3-Carboxy-4-methyl-5-propyl-2-furanpropanoic acid (CMPF) is a furan-fatty acid whose level appears to be elevated in the blood of patients with T2DM and GDM compared to patients without diabetes. Furan-fatty acids are incorporated by phospholipids or cholesterol esters and are metabolized into dibasic urofuranic acids that also include CMPF, which are excreted in the urine $(8,9)$.

In mice, elevated CMPF levels cause glucose intolerance, inadequate insulin secretion, and decreased peripheral glucose utilization (10). Some of the mechanisms of action of CMPF are improper mitochondrial function in pancreatic $\beta$-cells, low ATP glucose storage, increased oxidative stress, dysfunction of cellular transcription mechanisms and, finally, decreased insulin secretion. As antioxidant treatments counteract the negative effects of CMPF on pancreatic $\beta$-cells, we could envisage future treatment of GDM (10).

Starting from the assumption that the onset of GDM occurs early in the very first weeks of pregnancy, the present study aimed to verify whether the biological markers tested (AN, L, AN/L and CMPF) can be associated with the diagnosis of GDM.

The main objective of this study was to propose a method for the early, first trimester diagnosis of GDM, which would include a panel of biomarkers associated with certain clinical and demographic parameters in the Caucasian population, considered to be at low risk of developing GDM. The secondary endpoint was to analyze the maternal-fetal complications associated with GDM.

\section{Patients and methods}

Study population. We conducted a prospective longitudinal study at the Obstetrics and Gynecology Clinic I, County Clinical Emergency Hospital of Cluj-Napoca, Romania, in the period between January 2018 and March 2019. The study included a total of 111 first trimester pregnant women.

The inclusion criteria were: Pregnant women aged 18-40 years, gestational age of 11-13 weeks +6 days singleton pregnancy, performance of an oral glucose tolerance test (OGTT) at 24-28 weeks of gestation (WG), compliance with the follow-up conditions, and delivery at the Obstetrics and Gynecology Clinic I of Cluj-Napoca.

All the procedures performed were in accordance with the ethical standards of the Institutional and National Research Ethics Committee and with the Declaration of Helsinki (1964) and its later amendments. Our report is based on the STROBE Statement (Strengthening the Reporting of Observational studies in Epidemiology) (11).

The exclusion criteria were: Patients known to be suffering from type 1 and 2 diabetes, with acute or chronic infectious pathology, multiple or intrauterine fetal death, pregnancies with chromosomal abnormalities or fatal fetal malformations, patients who did not comply with the follow-up conditions and those who refused to participate in the study.

The control group included 47 singleton pregnancy patients with physiological pregnancies who accepted to provide a blood sample at 11-13 weeks+6 days in order to determine L, AN and CMPF levels, with a normal OGTT result, and who gave birth at the Obstetrics and Gynecology Clinic I of Cluj-Napoca.

Structured questionnaires and hospital medical records provided information regarding maternal age, ethnicity, height, pre-pregnancy weight, smoking before pregnancy, reproductive, obstetrical and medical history during pregnancy and after delivery, and the APGAR score.

Blood collection and biochemical assays. Maternal blood samples were collected between 11 and $13+6$ weeks of gestation (WG) at the time of the combined first-trimester screening for aneuploidy, according to standardized surgical procedures. Venous blood samples were collected from peripheral vessels into commercially available Vacutainer CAT 6-ml PET tubes with clot activator to determine L, AN, and CMPF levels. The blood samples were centrifuged at 4,000 x g for $5 \mathrm{~min}$ after allowing the blood to clot for $30 \mathrm{~min}$ at room temperature, while maintained in a vertical position. Serum and plasma samples were stored at $-80^{\circ} \mathrm{C}$ until analysis. The diagnosis of gestational diabetes was set between 24-28 WG based on OGTTs and in compliance with the international recommendations of the International Association of the Diabetes and Pregnancy Study Groups criteria (IADPSG) (12). The OGTT was performed with $75 \mathrm{mg}$ of glucose intake (after $8 \mathrm{~h}$ of fasting). Gestational diabetes was diagnosed when one of the 3 values determined was altered: Fasting blood glucose $>92 \mathrm{mg} / \mathrm{dl}$ (5.1 mmol/l), 1-h glycemia >180 mg/dl (10 mmol/l), 2-h glycemia $>153 \mathrm{mg} / \mathrm{dl}(8.5 \mathrm{mmol} / \mathrm{l})$.

Serum CMPF, AN and L levels were determined using commercially available enzyme-linked immunosorbent assay (ELISA) kits. The concentrations of CMPF and of $\mathrm{AN}$ and $\mathrm{L}$ were determined according to the manufacturers' instructions included in the commercially available kits (MyBioSource, Inc. and Invitrogen/ThermoFisher Scientific, Inc., respectively).

Statistical analysis. Statistical analysis was performed using MedCalc Statistical Software version 19.1.5 (MedCalc Software by, Ostend, Belgium; https://www.medcalc.org; 2020). The continuous variable data were tested for normal distribution (Shapiro Wilk test) and were described by median and 25-75 percentiles. All quantitative variables had a non-normal distribution. The nominal data were characterized by frequency and percentage. Comparisons between groups were performed using the Man-Whitney or Chi-square test, whenever appropriate. Correlations between quantitative variables were verified using the Spearman's rank correlation coefficient. The cut-off value for $\mathrm{L}$ was calculated in order to differentiate between GDM and normal patients, using a receiver operating characteristic (ROC) curve. The independent association between variables and the presence of GDM were assessed by multivariate logistic regression. The model included the variables that achieved a P-value $<0.05$ 
Table I. Demographic characteristics of the study groups.

\begin{tabular}{|c|c|c|c|}
\hline Variables & GDM group $(\mathrm{N}=21)$ & Control group $(\mathrm{N}=47)$ & P-value \\
\hline Maternal age, years [mean (range)] & $32(30-35.5)$ & $30(27-33)$ & 0.030 \\
\hline Smoking, n (\%) & $7(33.3 \%)$ & $5(10.6 \%)$ & $\mathbf{0 . 0 3 0}$ \\
\hline $\operatorname{BMI}\left(\mathrm{kg} / \mathrm{m}^{2}\right)$ & $24.3(21.3 ; 28.4)$ & $20.9(19.8 ; 24.1)$ & 0.003 \\
\hline Family history of diabetes, $\mathrm{n}(\%)$ & $8(38.1 \%)$ & $8(17 \%)$ & 0.007 \\
\hline \multicolumn{4}{|l|}{ Parity, n (\%) } \\
\hline Nulliparous & $8(38.8 \%)$ & $31(66 \%)$ & 0.060 \\
\hline Multiparous & & & 0.070 \\
\hline Previous GDM & $3(14.28 \%)$ & - & \\
\hline Non-previous GDM & $10(47.61 \%)$ & $16(34 \%)$ & \\
\hline Previous macrosomia, $\%$ & $7(53.84 \%)$ & $3(18.7 \%)$ & NS \\
\hline
\end{tabular}

BMI, body mass index; GDM, gestational diabetes mellitus. NS, not significant. Significant P-values are noted in bold print.

Table II. Comparison of biochemical markers according to GDM status.

\begin{tabular}{|c|c|c|c|}
\hline Biochemical markers & GDM groupmedian $(25 ; 75$ percentiles $)$ & Control group median $(25 ; 75$ percentiles $)$ & P-value \\
\hline Adiponectin (AN) & $26.8(17.6 ; 70.1)$ & $28.4(18.2 ; 50.79)$ & 0.800 \\
\hline Leptin (L) & $32.7(25 ; 48.1)$ & $16.8(9.5 ; 32)$ & $<0.001$ \\
\hline $\mathrm{AN} / \mathrm{L}$ & $0.88(0.58 ; 2.9)$ & $1.42(0.89 ; 7.4)$ & $\mathbf{0 . 0 3 0}$ \\
\hline CMPF & $180.6(154.4 ; 201.9)$ & $179.2(153.1 ; 213.1)$ & 0.900 \\
\hline
\end{tabular}

GDM, gestational diabetes mellitus; AN/L, adiponectin/leptin ratio; CMPF, 3-carboxy-4-methyl-5-propyl-2-furanpropanoic acid. Significant P-values are noted in bold print.

in the univariate analysis. A P-value $<0.05$ was considered statistically significant.

\section{Results}

Out of the selected 111 patients, 68 patients underwent OGTT and were able to be monitored until delivery. The rest of the patients included in the study did not meet the follow-up criteria (43 were excluded from the study: 25 patients gave birth in others hospitals and 18 patients were lost during follow-up). Twenty-one patients were diagnosed with GDM based on the test results and 47 patients were non-GDM and were selected as a control group. The prevalence of GDM in the study group was $18.91 \%$ (21 patients confirmed with GDM/111 cases). The demographic data of the groups are summarized in Table I. The age of the patients with GDM was significantly more advanced than that of the patients who did not develop diabetes, and they also had a higher pre-pregnancy $\mathrm{BMI}(\mathrm{P}=0.030$ and $\mathrm{P}=0.003$, respectively). Smokers were more likely to develop GDM $(\mathrm{P}=0.030)$. The group of patients with GDM included more multiparous women (61.9\% compared to $34 \%$ in the control group), especially women who had previously developed GDM in a previous pregnancy. This group also included a higher percentage of patients with fetus macrosomia in previous pregnancies (53.8\% in GDM group vs. $18.7 \%$ in control group) (Table I).
Patients who developed GDM had significantly higher levels of L compared with those who did not develop diabetes $(\mathrm{P}<0.001)$. AN levels did not differ significantly between the patient groups. The AN/L ratio was significantly lower in the patients with GDM $(\mathrm{P}=0.030)$. We did not find significant values of CMPF in patients with GDM compared to those in the control group (Table II).

The L cut-off value we calculated was $16 \mathrm{ng} / \mathrm{ml}$. The probability of developing GDM was higher in the case of patients with levels above this cut-off value [area under the curve $(\mathrm{AUC})=0.775,95 \%$ confidence interval (CI), 0.658-0.867], sensitivity $100 \%$ (95\% CI, 83.9-100), specificity $48.9 \%$ (95\% CI, 34.1-63.9) $(\mathrm{P}<0.001)$ (Fig. 1).

In order to ascertain which variables independently predict the onset of GDM, we constructed a model using logistic multivariate regression. We introduced the statistically significant variables that were associated with GDM in the univariate analysis. Patients of advanced maternal age and higher L levels had a 1.16 and 1.06 time higher risk, respectively, of developing GDM (Table III).

There was a weak negative correlation between $\mathrm{AN}$ values and newborn weight and a weak positive correlation with the initial weight of the mother. AN/L coefficient was moderately correlated with newborn weight (Table IV).

In patients diagnosed with GDM, the rate of obstetric complications, such as preterm birth, premature rupture of 
Table III. Multivariate logistic regression for the presence of GDM.

\begin{tabular}{lcccccc}
\hline & & & & \multicolumn{2}{c}{$\begin{array}{c}95 \% \\
\text { for OR }\end{array}$} \\
\cline { 5 - 7 } & & & & & & \\
\cline { 5 - 7 } & & & P-value & OR & Min & Max \\
Maternal age & 0.15 & 0.050 & 1.16 & 1.00 & 1.36 \\
Pre-pregnancy BMI & 0.05 & $\mathbf{0 . 5 0 0}$ & 1.05 & 0.88 & 1.26 \\
Smoking & 0.39 & 0.600 & 1.48 & 0.31 & 7.07 \\
Leptin & 0.06 & $\mathbf{0 . 0 2 0}$ & 1.06 & 1.00 & 1.11 \\
\hline
\end{tabular}

BMI, body mass index; GDM, gestational diabetes mellitus; OR, odds ratio; CI, confidence interval. Significant P-values are noted in bold print.

Table IV. Correlations for newborn weight and APGAR score.

\begin{tabular}{|c|c|c|c|c|}
\hline & \multicolumn{2}{|c|}{ Newborn weight } & \multicolumn{2}{|c|}{ APGAR score } \\
\hline & $\mathrm{R}$ & P-value & $\mathrm{R}$ & P-value \\
\hline Maternal age & 0.024 & 0.800 & 0.088 & 0.400 \\
\hline Pre-pregnancy BMI & 0.280 & 0.020 & 0.030 & 0.800 \\
\hline Adiponectin & -0.289 & 0.020 & -0.014 & 0.900 \\
\hline Leptin & 0.175 & 0.100 & -0.048 & 0.600 \\
\hline $\mathrm{AN} / \mathrm{L}$ & -0.332 & 0.007 & -0.049 & 0.700 \\
\hline CMPF & 0.102 & 0.400 & -0.215 & 0.070 \\
\hline
\end{tabular}

BMI, body mass index; AN/L, adiponectin/leptin ratio; CMPF, 3-carboxy-4-methyl-5-propyl-2-furanpropanoic acid. Significant P-values are noted in bold print.

membranes (PROM), hydramnios, dystocia of the shoulders, cervical or perineal lacerations, was higher compared to the control group, but only polyhydramnios presented with a significant difference. In addition, the rate of Cesarean section $(\mathrm{P}<0.001)$, the number of cases of fetal macrosomia $(\mathrm{P}=0.01)$ and the frequency of hypoglycemia in newborns $(\mathrm{P}=0.030)$ was higher in the GDM group compared to the control group. Newborns born to mothers in the GDM group, who required neonatal intensive care unit (NICU) admission were more numerous than those of the non-diabetic mothers $(\mathrm{P}=0.040)$ (Table V).

\section{Discussion}

The present study demonstrated that $\mathrm{L}$ levels and AN/L ratio can predict GDM development as early as the first trimester of pregnancy, alone or in combination with demographic parameters such as age, smoking, or pre-pregnancy body mass index (BMI), while AN and 3-carboxy-4-methyl-5-propyl-2-furanpropanoic acid (CMPF) levels do not. Elevated L levels proved to be a good predictor of GDM. In addition, the $\mathrm{AN} / \mathrm{L}$ ratio was found to be significantly correlated with the

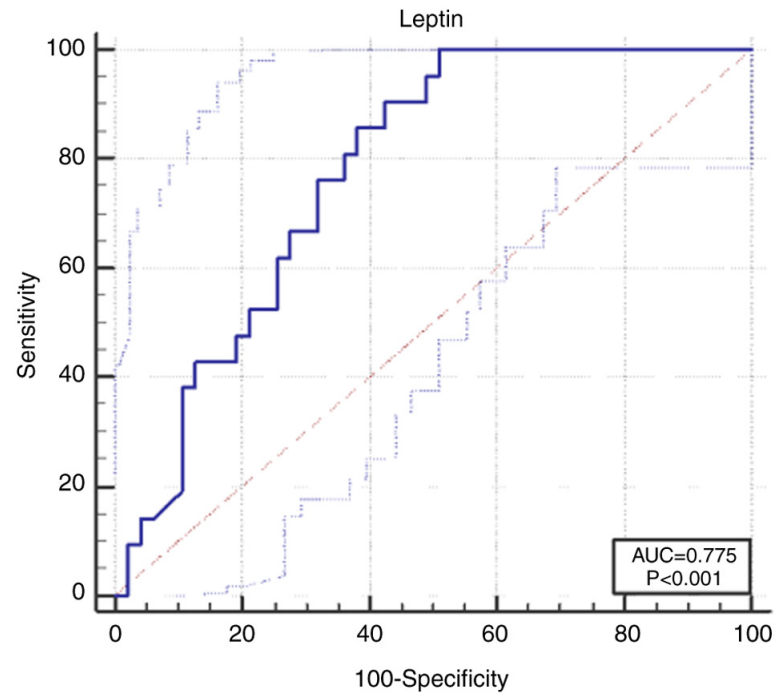

Figure 1. ROC curve for leptin. ROC, receiver operating characteristic; AUC, area under the ROC curve.

development of GDM. However, in our research, AN and CMPF levels were not associated with GDM. The results were independent of age, BMI and smoking habits. Elevated $\mathrm{L}$ levels were found in all patients with GDM, regardless of age. The more advanced the age of the patients, the higher the frequency of GDM when several risk factors were accumulated. Nonetheless, the changes in L levels and the AN/L ratio occurred independently of age. The logistic regression (Table III) showed that the independent nature of the variables that were introduced in the model, was preserved.

In our study, L levels were elevated as early as the first trimester in pregnant women who developed GDM, independently of age, pre-pregnancy BMI and smoking habits $(\mathrm{OR}=1.16)$. Moreover, there was a direct correlation between elevated L levels and the risk of developing GDM [AUC 0.775 (95\% CI, 0.658-0.867), sensitivity 100\% (95\% CI, 83.9-100), specificity $48.9 \%$ (95 \% CI 34.1-63.9), $\mathrm{P}<0.001]$.

The strong point of this study consists in the way in which the patients were selected. OGTT was performed in all patients included in the study, unlike in other studies, in which OGTT was performed only in patients with risk factors. Thus, we avoided overdiagnosis in the monitored groups and implicitly the highlighting of some biased AN, L, AN/L and CMPF values (13).

It is also important to note that this study was conducted on a Caucasian population, with a very low rate of diabetes compared to the black population $(14,15)$. These results are consistent with those of a meta-analysis published by Bao et al which concluded that $\mathrm{L}$ levels in the first trimester or in the early second trimester were $7.25 \mathrm{ng} / \mathrm{ml}$ higher $(95 \% \mathrm{CI}$ 3.27-11.22), among women who later developed GDM than women who did not (16).

The results obtained in the present study are confirmed by other studies. In a study performed on 47 pregnant women Qiu et al obtained a GDM frequency rate of 5.7\% (18.9\% in our study). The increase in $L$ levels in the first trimester were found to be highly significant for the development of GDM $(\mathrm{P}<0.001)$. An increase in L levels above $31.0 \mathrm{ng} / \mathrm{ml}$ resulted in a 4.7 times 
Table V. Peripartum parameters in the GDM and control groups.

\begin{tabular}{|c|c|c|c|}
\hline & GDM group $(\mathrm{N}=21)(\%)$ & Control group $(\mathrm{N}=47)(\%)$ & P-value \\
\hline \multicolumn{4}{|c|}{ Maternal complications, n (\%) } \\
\hline PROM & $2(9.52)$ & $2(4.25)$ & \\
\hline Preterm labor & $2(9.52)$ & $1(2.12)$ & \\
\hline Polyhydramnios & $7(33.3)$ & - & $<0.001$ \\
\hline Postterm pregnancy & - & $2(4.25)$ & \\
\hline Shoulder dystocia & $2(9.52)$ & & NS \\
\hline Lacerations & $2(9.52)$ & $1(2.12)$ & NS \\
\hline Fetal dystocia & $4(19.1)$ & $5(10.63)$ & NS \\
\hline \multicolumn{4}{|c|}{ Fetal complications, n (\%) } \\
\hline IUGR & $1(4.76)$ & - & NS \\
\hline Perinatal asphyxia & $1(4.76)$ & $1(2.12)$ & NS \\
\hline \multicolumn{4}{|l|}{ Mode of delivery, n (\%) } \\
\hline Vaginal birth & $7(33.3)$ & $32(68)$ & $<0.001$ \\
\hline Cesarean section & $14(66.6)$ & $15(32)$ & \\
\hline \multicolumn{4}{|l|}{ Newborn gender, n (\%) } \\
\hline Male & $10(47.6)$ & $22(46.8)$ & NS \\
\hline Female & $11(52.3)$ & $23(53.2)$ & \\
\hline \multicolumn{4}{|l|}{ Birth weight (g) } \\
\hline$\geq 2,500$ & $2(9.52)$ & $1(2.12)$ & 0.040 \\
\hline $2,500-3,900$ & $11(52.38)$ & $41(87.23)$ & 0.010 \\
\hline$\leq 3,900$ & $8(38.1)$ & $5(10.6)$ & \\
\hline \multicolumn{4}{|l|}{ APGAR score, n (\%) } \\
\hline$\geq 7$ & $1(4.76)$ & $1(2.12)$ & NS \\
\hline$<7$ & $20(95.23)$ & $46(97.87)$ & \\
\hline \multicolumn{4}{|c|}{ Neonatal morbidity, n (\%) } \\
\hline Monitoring in NICU & $7(33.3)$ & $6(12.76)$ & 0.040 \\
\hline Hypoglycemia & $6(28.57)$ & $1(2.12)$ & 0.030 \\
\hline
\end{tabular}

GDM, gestational diabetes mellitus; PROM, premature rupture of membranes; IUGR, intrauterine growth restriction; NICU, neonatal intensive care unit. NS, not significant. Significant P-values are noted in bold print.

increase in the risk of developing GDM (95\% CI, 1.2-18.0). At the same time, other authors found a close linear increased association between L levels and the risk of developing GDM. Thus, a $10 \mathrm{ng} / \mathrm{m}$ increase in leptin levels was followed by a $20 \%$ increase in the risk of developing GDM (17). In our study, $\mathrm{L}$ values that were double compared to those in the control group were associated with a $16 \%$ increased risk in GDM.

While conducting a study in which only pregnant women at risk for GDM underwent OGTT, Thagaard et al reported an increase in L levels only in obese patients with GDM and not in normal weight patients (13). In the present research, the low AN/L ratio showed a statistically significant correlation with the development of GDM and fetal macrosomia. Thagaard et al also reported that alterations of this ratio in the first trimester of pregnancy have a good predictive value for GDM in patients with normal weight or moderate obesity $\left(\mathrm{BMI}<35 \mathrm{~kg} / \mathrm{m}^{2}\right)$ (13). Skvarca et al showed that the AN/L ratio is the best marker for assessing insulin resistance in normal weight pregnant women, being correlated with the HOMA-IR index (6).
Various studies have reported maternal obesity as the leading cause of increased L levels in pregnant women with GDM. In this case, elevated L levels would be the result of changes due to obesity combined with pregnancy-related physiological changes, and not the result of independent changes due to GDM. In normal-weight pregnant women GDM is caused by inadequate insulin synthesis, while in overweight/obese pregnant women, GDM is the result of inadequate insulin synthesis and increased peripheral insulin resistance (15-22).

Therefore, increased $\mathrm{L}$ levels are the result of the above mentioned changes and obese patients may develop peripheral resistance to $\mathrm{L}$, similar to insulin resistance. However, the mechanisms involved in the correlation between L levels, peripheral insulin resistance, insulin levels and obesity during pregnancy, are still incompletely elucidated and represent a current study issue of international interest on family health (23).

Our study did not show statistically significant associations between AN levels and GDM development; the values being 
similar between the GDM and the non-GDM group (26.8 vs. $28.4 \mathrm{~g} / \mathrm{ml}$ ). Therefore, AN does not predict GDM development. Instead, our research showed an inversely proportional association between AN levels and the weight of newborns at birth $(\mathrm{P}=0.020)$. This result was also confirmed by Nanda et al, who showed the role of hypoadiponectinemia in predicting fetal macrosomia (24).

Studies on the connection between the decrease in AN levels in pregnancy and the development of GDM are controversial. Many of these studies could not find a correlation between changes in AN levels in the first trimester and the development of GDM, similarly to our findings (6,25-27).

Another study reported a positive correlation between AN levels and fetal weight at birth, as well as a negative correlation between AN levels and head circumference (28). Paradisi et al reported a 5\% physiological decrease in AN levels during pregnancy in the second trimester of pregnancy compared to the first trimester, as well as a $20 \%$ decrease in AN levels in the third trimester, compared to the first trimester, which was not due to GDM. However, given that AN level variations in the first trimester in pregnant women with GDM were not significantly altered compared to non-GDM patients, this biomarker did not prove to be effective as a predictive factor for GDM (29).

On the other hand, some studies have shown that AN levels are decreased in GDM, highlighting that this decrease in AN levels is independent of maternal adiposity and could be predictive of GDM development $(17,30,31)$.

Another biomarker of interest when trying to explain the changes leading to the development of GDM is CMPF, a metabolite of furan whose level is elevated in the plasma of patients with T2DM and in pregnant women with GDM. Our research did not reveal significant differences between the two groups (GDM and non-GDM group) in as far as this metabolite is concerned [mean (25; 75 percentiles): 180.6 $(154.4 ; 201.9)$ vs. $179.2(153.1 ; 213.1)]$. Lankinen et al showed that elevated CMPF levels may be the result of a diet rich in fish, and that elevated levels are not associated with the development of GDM (32). However, further studies on a large number of cases are needed to demonstrate the relationship between the increase in CMPF levels and the development of GDM.

The limitations of this study consist, first of all, in the small number of patients, the short time allotted to the selection of cases, and the number of samples collected during pregnancy.

The markers (L, AN and CMPF) were determined at the same time with the genetic screening, and there may be variations depending on various factors (fasting prior to sample collection or not). Multiple harvests during pregnancy would be needed in order to clarify how these markers change with the evolution of the pregnancy.

A second issue includes the determination of the way in which BMI can influence L and AN levels or, in other words, to what extent $\mathrm{L}$ and $\mathrm{AN}$ levels are altered by the presence of GDM and/or by obesity in patients with a high BMI.

Continuing this research on large groups of patients could help physicians select patients at risk to develop GDM, open new horizons in as far as the therapeutic conduct in the case of these patients is concerned, and provide a better understanding of the pathophysiological mechanisms of this disease.
The use of biomarkers for the early diagnosis of GDM can have a beneficial impact on maternal health decreasing the mortality rate and maternal-fetal morbidity by changing lifestyle, diet, and early treatment.

In conclusion, our results concerning $\mathrm{L}$ values are encouraging and predictive of the development of GDM as early as the first trimester of pregnancy. Most importantly, this parameter is independent of the patient BMI, contrary to what many other studies report. A low AN/L ratio value is predictive of GDM development and is associated with fetal macrosomia. Research on far larger study groups is needed to demonstrate the predictive role of CMPF and AN.

\section{Acknowledgements}

Not applicable.

\section{Funding}

This study was partially funded by the 'Iuliu Hatieganu' University of Medicine and Pharmacy of Cluj-Napoca, Romania for Doctoral Research Projects (no. 1300/48/13.01.2017).

\section{Availability of data and materials}

The datasets used and/or analyzed in the present study are available from the corresponding author on reasonable request.

\section{Authors' contributions}

ARF was the main coordinator of the project and was responsible for the study design. GC and ARF drafted the manuscript of the present paper. RMP was involved in the supervising of the data collection and stratification. AS contributed to data assembly and analysis. SF and MD contributed to the study design and manuscript revision. All authors contributed intellectually to this manuscript and have approved this final version.

\section{Ethics approval and consent to participate}

The study was approved by the Bioethics Commission of the 'Iuliu Hatieganu' University of Medicine and Pharmacy in Cluj-Napoca, Romania (Nr.247/08.06.2017). Patients who participated in this research had complete clinical data. Signed informed consents were obtained from the patients or the guardians.

\section{Patient consent for publication}

Not applicable.

\section{Competing interests}

The authors declare that they have no competing interests.

\section{References}

1. Soheilykhah S, Mojibian M, Rahimi-Saghand S, Rashidi M and Hadinedoushan $\mathrm{H}$ : Maternal serum leptin concentration in gestational diabetes. Taiwan J Obstet Gynecol 50: 149-153, 2011. 
2. Moini J: Chapter 6: The Health Impact of Diabetes. In Epidemiology of Diabetes. Elsevier, pp115-145, 2019.

3. Sacks DA, Hadden DR, Maresh M, Deerochanawong C, Dyer AR, Metzger BE, Lowe LP, Coustan DR, Hod M, Oats JJ, et al: Frequency of gestational diabetes mellitus at collaborating centers based on IADPSG consensus panel-recommended criteria: The hyperglycemia and adverse pregnancy outcome (HAPO) study. Diabetes Care 35: 526-528, 2012.

4. Ornoy A, Reece EA, Pavlinkova G, Kappen C and Miller RK: Effect of maternal diabetes on the embryo, fetus, and children: Congenital anomalies, genetic and epigenetic changes and developmental outcomes. Birth Defects Res C Embryo Today 105: 53-72, 2015.

5. Standards of Medical Care in Diabetes-2016: Summary of revisions. Diabetes Care 39 (Suppl 1): S4-S5, 2016.

6. Skvarca A, Tomazic M, Blagus R, Krhin B and Janez A: Adiponectin/leptin ratio and insulin resistance in pregnancy. J Int Med Res 41: 123-128, 2013.

7. Li H, Dong A and Lv X: Advanced glycation end products and adipocytokines and oxidative stress in placental tissues of pregnant women with gestational diabetes mellitus. Exp Ther Med 18: 685-691, 2019.

8. Prentice KJ, Luu L, Allister EM, Liu Y, Jun LS, Sloop KW Hardy AB, Wei L, Jia W, Fantus IG, et al: The furan fatty acid metabolite CMPF is elevated in diabetes and induces $\beta$ cell dysfunction. Cell Metab 19: 653-666, 2014.

9. Diaz SO, Pinto J, Graç G, Duarte IF, Barros AS, Galhano E, Pita C, Almeida Mdo C, Goodfellow BJ, Carreira IM and Gil AM: Metabolic biomarkers of prenatal disorders: An exploratory NMR metabonomics study of second trimester maternal urine and blood plasma. J Proteome Res 10 3732-3742, 2011.

10. Nolan CJ: Lipotoxicity, $\beta$ cell dysfunction, and gestational diabetes. Cell Metab 19: 553-554, 2014.

11. von Elm E, Altman DG, Egger M, Pocock SJ, Gøtzsche PC and Vandenbroucke JP; STROBE Initiative: The strengthening the reporting of observational studies in epidemiology (STROBE) Statement: Guidelines for reporting observational studies. PLoS Med 16: e296, 2007.

12. International Association of Diabetes and Pregnancy Study Groups Consensus Panel; Metzger BE, Gabbe SG, Persson B Buchanan TA, Catalano PA, Damm P, Dyer AR, Leiva Ad, Hod M, et al: International Association of Diabetes and Pregnancy Study Groups recommendations on the diagnosis and classification of hyperglycemia in pregnancy. Diabetes Care 33 676-682, 2010

13. Thagaard IN, Krebs L, Holm JC, Lange T, Larsen T and Christiansen M: Adiponectin and leptin as first trimester markers for gestational diabetes mellitus: A cohort study. Clin Chem Lab Med 55: 1805-1812, 2017.

14. Savvidou M, Nelson SM, Makgoba M, Messow CM, Sattar N and Nicolaides K: First-trimester prediction of gestational diabetes mellitus: Examining the potential of combining maternal characteristics and laboratory measures. Diabetes 59: 3017-3022, 2010

15. Hedderson MM, Xu F, Darbinian JA, Quesenberry CP, Sridhar S, Kim C, Gunderson EP and Ferrara A: Prepregnancy SHBG concentrations and risk for subsequently developing gestational diabetes mellitus. Diabetes Care 37: 1296-1303, 2014.

16. Bao W, Baecker A, Song Y, Kiely M, Liu S and Zhang C: Adipokine levels during the first or early second trimester of pregnancy and subsequent risk of gestational diabetes mellitus: A systematic review. Metabolism 64: 756-764, 2015

17. Qiu C, Williams MA, Vadachkoria S, Frederick IO and Luthy DA: Increased maternal plasma leptin in early pregnancy and risk of gestational diabetes mellitus. Obstet Gynecol 103: 519-525, 2004

18. Maple-Brown L, Ye C, Hanley AJ, Connelly PW, Sermer M, Zinman B and Retnakaran R: Maternal pregravid weight is the primary determinant of serum leptin and its metabolic associations in pregnancy, irrespective of gestational glucose tolerance status. J Clin Endocrinol Metab 97: 4148-4155, 2012.
19. Eriksson B, Löf M, Olausson $\mathrm{H}$ and Forsum E: Body fat, insulin resistance, energy expenditure and serum concentrations of leptin, adiponectin and resistin before, during and after pregnancy in healthy Swedish women. Br J Nutr 103: 50-57, 2010.

20. Misra VK and Trudeau S: The influence of overweight and obesity on longitudinal trends in maternal serum leptin levels during pregnancy. Obesity (Silver Spring) 19: 416-421, 2011.

21. Cruciat G, Nemeti G, Goidescu I, Anitan S and Florian A: Hypertriglyceridemia triggered acute pancreatitis in pregnancy-diagnostic approach, management and follow-up care. Lipids Health Dis 19: 2, 2020.

22. Park S, Kim MY, Baik SH, Woo JT, Kwon YJ, Daily JW, Park YM, Yang JH and Kim SH: Gestational diabetes is associated with high energy and saturated fat intakes and with low plasma visfatin and adiponectin levels independent of prepregnancy BMI. Eur J Clin Nutr 67: 196-201, 2013.

23. Froy O, Sherman H, Bhargava G, Chapnik N, Cohen R, Gutman R, Kronfeld-Schor N and Miskin R: Spontaneous caloric restriction associated with increased leptin levels in obesity-resistant amUPA mice. Int J Obes 35: 226-235, 2011.

24. Nanda S, Akolekar R, Sarquis R, Mosconi AP and Nicolaides KH: Maternal serum adiponectin at 11 to 13 weeks of gestationin the prediction of macrosomia. Prenat Diagn 31: 479-483, 2011.

25. Saucedo R, Zarate A, Basurto L, Hernandez M, Puello E, Galvan R and Campos S: Relationship between circulating adipokines and insulin resistance during pregnancy and postpartum in women with gestational diabetes. Arch Med Res 42: 318-323, 2011.

26. Yamamoto Y, Hirose H, Saito I, Tomita M, Taniyama M, Matsubara K, Okazaki Y, Ishii T, Nishikai K and Saruta T: Correlation of the adipocyte-derived protein adiponectin with insulin resistance index and serum high-density lipoprotein-cholesterol, independent of body mass index, in the Japanese population. Clin Sci 103: 137-142, 2002.

27. Mohammadi T and Paknahad Z: Adiponectin concentration in gestational diabetic Women: A case-control study. Clin Nutr Res 6: 267-276, 2017.

28. Vernini JM, Moreli JB, Antônio RA, Costa RA, Negrato CA, Rudge MV and Calderon IM: Maternal adipokines and insulin as biomarkers of pregnancies complicated by overweight and obesity. Diabetol Metab Syndr 8: 68, 2016.

29. Paradisi G, Ianniello F, Tomei C, Bracaglia M, Carducci B, Gualano MR, La Torre G, Banci M and Caruso A: Longitudinal changes of adiponectin, carbohydrate and lipid metabolism in pregnant women at high risk for gestational diabetes. Gynecol Endocrinol 26: 539-545, 2010.

30. Lain KY, Daftary AR, Ness RB and Roberts JM: First trimester adipocytokine concentrations and risk of developing gestational diabetes later in pregnancy. Clin Endocrinol (Oxf) 69: 407-411, 2008.

31. Lacroix M, Battista MC, Doyon M, Ménard J, Ardilouze JL, Perron P and Hivert MF: Lower adiponectin levels at first trimester of pregnancy are associated with increased insulin resistance and higher risk of developing gestational diabetes mellitus. Diabetes Care 36: 1577-1583, 2013.

32. Lankinen MA, Hanhineva K, Kolehmainen M, Lehtonen M, Auriola S, Mykkänen H, Poutanen K, Schwab U and Uusitupa M: CMPF does not associate with impaired glucose metabolism in individuals with features of metabolic syndrome. PLoS One 10: e0124379, 2015.

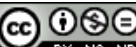

This work is licensed under a Creative Commons Attribution-NonCommercial-NoDerivatives 4.0 International (CC BY-NC-ND 4.0) License. 